\title{
Conversion of HI Molecule to $\mathrm{H}_{2}$ Molecule
}

\author{
Arjun Kumar Gautam \\ Department of Physics, Mahendra Multiple Campus, Nepalgunj
}

\begin{abstract}
In this article I review the historical development and conversion of atomic to molecular hydrogen in astronomy. I discuss how the discoveries of $\mathrm{HI}$ and $\mathrm{H}_{2}$ in the interstellar medium were followed by studies of the relative abundance of atomic and molecular gas. Understanding this led to increasingly sophisticated theoretical models for $\mathrm{H}_{2}$ formation on the surface of interstellar dust grains. In certain situations, astronomical data can be used to constrain the formation rate of $\mathrm{H}_{2}$ molecules. Finally, I use the reasonably well-determined chemistry of $\mathrm{HI}$ and $\mathrm{H}_{2}$ to determine the overall timescale of star formation.
\end{abstract}

Keywords: $\mathrm{HI}$ Molecule, $\mathrm{H}_{2}$ Molecule, interstellar dust grain, time scale, hyperfine transition, theoretical model

\section{INTRODUCTION :}

Neutral atomic hydrogen is a normal, electrically neutral hydrogen atom with one proton and one electron. It is commonly referred to as HI (pronounced $\mathrm{H}$-one), and is located throughout galaxies as HI clouds or external to galaxies as part of the inter cloud gas. It is detected via the spin-flip transition at $21 \mathrm{~cm}$ in the radio, and $\mathrm{HI}$ clouds were used to determine the structure of our Galaxy from our location within it. Atomic hydrogen (HI) is the most abundant atom in the universe and $\mathrm{H}_{2}$ the most abundant molecule. The importance of these two species and their obvious inter-connection has led to an ongoing interest in their relationship in a wide variety of astrophysical situations. My focus here is on the formation of $\mathrm{H}_{2}$ in the context of evolution of material from more diffuse, atomic regions, to denser, primarily molecular clouds of gas, which is the initiation of the process of star formation.

\section{DETECTION OF ATOMIC AND}

\section{MOLECULAR HYDROGEN IN THE}

\section{INTERSTELLAR MEDIUM}

The possibility of observing HI through its $21 \mathrm{~cm}$ hyperfine transition was predicted by van der Hulst (1945) and by Shklovski (1948). These papers were strong motivations for experimental searches. Different groups had near-simultaneous success a few years later (Ewen \& Purell 1951, Muller \& Oort 1951, and report by Pawsey 1951). The work of the Harvard group was the extraordinary $\mathrm{PhD}$ research of $\mathrm{H}$. Ewen, who pursued this effort despite his expectation that only an upper limit would result (Ewen 2007). The initial detection of HI was very quickly followed up by large-scale studies of HI in the Milky Way, and the value of $21 \mathrm{~cm}$ astronomy was quickly established.

Initial work suggested that dust and atomic hydrogen densities are proportional even in clouds with modest visual extinction(Tdust $\leq 3$; Lilley 1955). This correlation was, however, found to break by Bok, Lawrence, \& Menon (1955). In a very early study of the outer galaxy, van de Hulst, Muller \& Oort (1954) found only $30 \%$ of the HI intensity in Taurus expected from dust obscuration, and attributed this to conversion of hydrogen into molecular form. In a more detailed study of the same region, Garzoli \& Varsavsky (1966) found that there was an inverse correlation between HI column density and visual extinction. Mészáros (1968) found that the column density of atomic hydrogen in the dust cloud near $\rho$ Ophiuchi was essentially independent of dust column density and possibly decreased along lines of sight having the greatest column density of dust. He also suggested that the hydrogen is being converted to molecular form in this dense regions.

Following the remarkably early discussions about the formation and importance of $\mathrm{H}_{2}$ (van de Hulst 1948 and retrospective discussion in van de Hulst 1997), sophisticated theories of $\mathrm{H}_{2}$ formation on grains were developed in the 1960's (McCrea \& McNally 1963, Gould \& Salpeter 1963). These undoubtedly encouraged the search for $\mathrm{H}_{2}$ which is, however, extremely difficult to observe in the general 
interstellar medium due to lack of energy levels with appreciable population at low temperatures, and the weak transitions between rotational levels of the homonuclear molecule. The challenging experinmental prospect and the importance of $\mathrm{H}_{2}$ were both discussed in detail by Field, Somerville, \&Dressler (1966).

$\mathrm{H}_{2}$ was first detected by Carruthers (1970), after an earlier unseccessful attempt (Carruthers 1967; see Caruthers 1969 for a description for equipment). These initial rocket observations showed that towards the star $\varsigma$ Per the atomic and molecular column densities were approximately $4 \times 10^{20} \mathrm{~cm}^{-2}$. This work was soon followed by Copernicus satellite studies showing that a significant fraction of the interstellar gas in the direction of many stars with color excess $\geq 0.1$ mag was molecular, while only a very small fraction of the gas was molecular for unreddened stars (Spitzer etal. 1973).

Tracing $\mathrm{H}_{2}$ directly through UV absorption in regions of greater column density is difficult because the dust extinction at UV wavelengths results in severe attenuation of background sources. Infrared rotation-vibration lines have been utilized to a limited extent(Lacy et al. 1994). The pure rotational lines of $\mathrm{H}_{2}$ are at longer wavelengths (up to $\lambda=28$ $\mu \mathrm{m})$, but they still require temperatures of over 100 $\mathrm{K}$ to be seen in emission. This means that in a general interstellar medium they are not detected. Results have been obtained on selected nearby regions with higher temperatures(e.g. Neufeld et al. 2006) and suprisingly strong emission has been detected from certain galaxies (Applenton et al.2006).

In general, astronomers assume that the $\mathrm{H}_{2}$ in well-shielded regions having Av 1 mag is entirely molecular, and typically referance abundances of other molecular species to an inferred, albeit unobserved column density of $\mathrm{H}_{2}$.

\section{THEORETICAL MODELS OF $\mathrm{H}_{2}$ FORMATION} Many experts in this field have attended to present the state of the art in theoretical calculations for formation of $\mathrm{H}_{2}$ on the surfaces of dust grains. I here only broadly review the historical development of this subject. I shall not discuss $\mathrm{H}_{2}$ formation by purely gas phase reactions. For formation of $\mathrm{H}_{2}$ at low densities in the early universe, reactions involving $\mathrm{H}_{2}$ and $\mathrm{H}_{2}$ are dominant (Galli \& Palla 1998). At higher densities, three body reactions become more important (Palla et al. 1983), and these are critical for production of $\mathrm{H}_{2}$ during the formation of the first stars in the universe (Yoshida et al. 2006).

As mentioned above, detailed theoretical modeling of $\mathrm{H}_{2}$ formation on dust grains dates back to at least the 1960's with the paper by McCrea \& McNally (1963), which even included a grain size distribution. The approach adopted was to use a rate equation to describe the changes in the average properties of a volume of an interstellar cloud. It was assumed that an $\mathrm{H}$ atom in the gas phase sticks to a grain which it hits. It is also assumed that there is at least one $\mathrm{H}$ atom on the surface of a grain, so that when a hydrogen atom hits it and sticks, the two atoms will recombine to form a $\mathrm{H}_{2}$ molecule, with sufficient energy released to desorb the newlyformed molecule from the grain. The number of $\mathrm{H}_{2}$ molecules formed per unit time per unit time per unit volume of the cloud is proportional to the number density of hydrogen atoms, $\mathrm{n}(\mathrm{HI})$ and of grains, $\mathrm{ng}$. However, the number of grains is proportional to the total proton density $n_{0}$. If we define $n_{1}=n(H I)$, $\mathrm{n}_{0}=\mathrm{n}_{1}+2 \mathrm{n}_{2}$, and we can write

$$
\frac{d n_{z}}{d t} \dot{=} \dot{\mathrm{F}} \dot{\mathrm{n}}_{1} \dot{\mathrm{n}}_{0},
$$

where $\mathrm{F}$ is the formation rate constant, having units $\mathrm{cm}^{3} \mathrm{~s}^{-1}$. It includes a number of factors, including the probability that the incident atom will stick to the grain, and the grain area per proton in the gas. In the simplest approximation, the only temperature dependence is due to the speed of the $\mathrm{H}$ atoms, and taking $\mathrm{T}=100 \mathrm{~K}, \mathrm{McC}$ rea and McNally derived $\mathrm{F}=7 \times 10^{-17} \mathrm{~cm}^{3} \mathrm{~s}^{-1}$.

The physics of the dust grain surface was analyzed in considerably more detail by Hollenbatch \& Salpeter 
(1971). These authors considered physisorption (attraction by Van der Waals forces) holding $\mathrm{H}$ atoms on the grain, as well as the atomic mobility, which is essential for two adsorb atoms to be able to interact and form a $\mathrm{H}_{2}$ molecule. They wrote the formation rate of $\mathrm{H}_{2}$ in a more detailed form

$$
\frac{d n_{z}}{d t} \cdot \frac{1}{2} \square<\mathrm{v}_{H}>\mathrm{ngn}_{1}\langle\sigma \mathrm{g}\rangle,
$$

where $\gamma$ is the sticking probability for incident $\mathrm{H}$ atoms, $\left\langle\bigsqcup_{H}>\right.$ is their metan thermal velocity, and $<\sigma g>$ is the average grain cross section.

This issue had been addressed earlier by Knapp et al. (1966), who pointed out that if there were only physisorption, temperatures above $8 \mathrm{~K}$ would result in the probability of having two atoms simultaneously on the grain being so low that efficient recombination would be impossible. These authors also indicated that chemical adsorption (resulting from formation of a chemical bond between the $\mathrm{H}$ atom and an atom on the grain surface) would improve the prospect for efficient $\mathrm{H}_{2}$ formation over a wide range of temperatures. Hollenbach \& Salpeter also suggested that a "semichemical" enchancement of the binding energy of the $\mathrm{H}$ atoms could increase the temperature range for efficient $\mathrm{H}_{2}$ formation.

Cazaux \& Tielens (2004) developed a grain model including both sites that can physisorb $\mathrm{H}$ atoms and those that can provide chemisorption. While the specific binding energies and the barrier height separating the two types of sites, are uncertain, this model has the welcome effect of broadening the temperature range of efficient $\mathrm{H}_{2}$ formation to cover from to cover from somewhat below $10 \mathrm{~K}$ to somewhat above $30 \mathrm{~K}$. While still not encompassing all regions in which efficient $\mathrm{H}_{2}$ formation is indicated, this does at least include dark clouds and the majority of giant molecular cloud material. Chang, Cuppen, \& Herbst (2005) studied "mixed" grain surfaces composed of carbon and olivine material, and found that the temperature range for efficient $\mathrm{H}_{2}$ formation was moderately increased over that for homogeneous surfaces, for which it was very small. An investigation of rough grains by Cuppen \& Herbst (2005) indicates that $\mathrm{H}_{2}$ formation can proceed at temperatures up to $50 \mathrm{~K}$, with this limit depending on the details of bonding to the non-planar surface structure. More realistic models in this vein are obviously called for as we learn more about the surface properties of real interstellar grains.

As indicated in eq. 3.2, the rate of $\mathrm{H}_{2}$ formation is also dependent on the sticking probability. This has received considerable attention over the years, and certainly depends on grain composition and morphology. Buch and Zhang (1991) calculated the sticking probability of $\mathrm{H}$ and $\mathrm{D}$ atoms on amorphous ice particles, finding it to be close to unity at temperatures of dense interstellar clouds ( $\mathrm{T} \leq 20 \mathrm{~K})$, but dropping at higher temperatures. This may not be representative of all grains types and sizes but gives some confidence that $\gamma$ is in general of order unity.

Using a rate equation to describe the evolution of cloud constituents has definite limitations in that it ignores the discrete nature of the atoms and molecules involved. While it may be valid in the limit of large atomic fluxes and atoms on a grain, it is evident that in the situation with very little atomic hydrogen in a cloud, there can be less than one hydrogen atom absorbed on a grain, so that it is not correct to assume that the rate derived for a much higher population of $\mathrm{H}$ atoms applies. Considerations of this sort have motivated a large numbers of investigators to develop models that deal with the discrete nature of particles more explicitly. These generally are more complex than rate equation models, and are difficult to describe in any convenient manner. A good review of this topic is given by Herbst, Chang, \& Cuppen (2005) which also has an extensive list of references.

\section{THE USE OF HI AND H TO DETERMINE} TIME SCALES IN INTERSTELLAR CLOUDS

As early as in 1968, it was suggested that the time-dependent conversion of atomic to molecular hydrogen could be used to determine an "age" for 
an interstellar cloud; Mészáros (1968) took this as the time since the cloud was last ionized by a hot star. Solomon \& Werner (1971) and Shu (1973) addressed the question of how the amount of $\mathrm{HI}$ in a "molecular" cloud could be used to constrain its evolutionary history.

The UV measurements referred to in sec. 2 measure atomic and molecular column densities along lines of sight towards hot stars with modest foreground extinctions ( few mag.). To probe the atomic to molecular hydrogen ratio in regions of greater extinction is possible in principle using the pure rotational transitions of $\mathrm{H}_{2}$, but these are so weak that the spectral features are not generally detectable with presently-available equipment. We are left with using surrogates to trace the column density of $\mathrm{H}_{2}$; these are generally isotopologues of carbon monoxide which are optically thin, namely ${ }^{13} \mathrm{CO}$ and $\mathrm{O}$.

For tracing the atomic gas, the situation is challenging because $21 \mathrm{~cm}$ line emission is so widespread. Observations detect emission with large line widths in almost all directions, which makes it difficult to associate a specific velocity feature with a certain region of space along the line of sight. One situation which is more favorable is locking for HI self-absorption. Such features in $21 \mathrm{~cm}$ spectra are common, and can result from temperature variations as a function of velocity (different clouds of gas), or from variations along the line of sight. One situation that has proven very useful is when we detect a very narrow absorption feature in the HI spectrum. The very narrow absorption features are found to be highly correlated in terms of nonthermal line width and spatial extent with optically thin carbon monoxide isotopologues (Li \& Goldsmith 2003; Goldsmith \& Li 2005). This facts together with the line widths themselves indicating very low temperatures, sometimes below $20 \mathrm{~K}$, suggest that the atomic gas producing the HI narrow selfabsorption (or HISA) features is mixed with the molecular gas in the well-shielded central regions of dense molecular clouds.

\section{SUMMARY}

The issue of $\mathrm{HI}$ to $\mathrm{H}_{2}$ conversion remains a critical one for astrophysics. Laboratory measurements, theoretical models, and astronomical data are being used together to understand the complex surface chemistry and radiative transfer issues involved. I can look forward to continued progress in all of these areas, which will allow me to use the atomic to molecular hydrogen abundance ratio as an importance diagnostic of the evolution of the interstellar medium.

\section{REFERENCES}

1. Appleton, P.N., Xu, K.C., Reach, W., Dopita, M.A., Gao, Y., Lu, N., Popescu, C.C., Sulentic, J.W., Tuffs, R.J., \& M.S. 2006, ApJ, 639, L51

2. Bok, B.J., Lawrence, R.S., \& Menon, T.K. 1955, Pub. Astr. Soc. Pacific, 67, 108

3. Buch, V. \& Zhang, Q. 1991, ApJ, 379, 647

4. Crruthers, G.R. 1967, ApJ, 148, L141

5. Carruthers, G.R. 1969, Appl. Optics, 8(3), 633

6. Crruthers, G.R. 1970, ApJ, 161, L81

7. Cazaux, S. \& Tielens, A.G.G.M 2004, ApJ, 604, 222

8. Chang, Q., Cuppen, H.M., \& Herbst, E. 2005, A\&A, 434, 599

9. Chang, Q., Cuppen, H.M., \& Herbst, E. 2006, A\&A, 458, 497

10. Ewen, H. \& Purcell E.M. 1951, Nature, 169, 356

11. Ewen, H. 2007, private communication

12. Field, G.H., Somerville, W.B., \& Dressler, K. 1966, Ann. Rev. Astr. Astrophys., 5, 207

13. Galli, D. \& Palla, F. 1998, A\&A, 335, 403

14. Garzoli, S. L. \& Varavsly 1966, ApJ, 145, 79

15. Gould, R.J. \& Salpeter, E.E. 1963, ApJ, 138,393

16. Herbst, E., Chang, Q., \& Cuppen, H.M. 2005, Chemistry an Interstellar Grains, in Journal of Physics Conference Series 6 (2005) Light, Dust and Chemical Evolution (:IOP Publishing), 18

17. Hollenbach, D. \& Salpeter, E.E. 1971, ApJ, 163, 155

18. Knapp, H.F.P., van den Meijdenberg, C.J.N., Beenakker, J.J.M, \& van de Hulst, H.C. 1966, B.A.N. 18,256 
19. Li, D. \& Goldsmith, P.F. 2003, ApJ, 585, 823

20. Lilley, A.E. 1955, ApJ, 121, 559

21. McCrea, W.H. \& Mcnally, D. 1960, MNRAS, 121,238

22. Mészáros, P. 1968, Astr.Sp.Sci., 2, 510

23. Muller, C.A. \& Oort, J,H. 1951, MNRAS, 121, 238

24. Neufeld, D.A., Melnick, G.J., Sonnentrucker, P., Bergin, E.A., Green, J.D., Kim, K.H., Watson, D.M., Forrest, W.J., \& Pipher, J.L. 2006, ApJ, 649, 816

25. Palla, F., Salpeter, E.E., \& Sathler, S.W. 1983, ApJ, 271, 632

26. Pawsey, J.L. 1951, Nature, 168, 357

27. Shklovski, I.S. 1948, Astron. Zh. SSSR, 26(1948) 1,10

28. Shu, F.H. On the Genetic Relation Between InterstellarClouds andDustClouds, in Interstellar Dust and Related Topics, ed. J.M. Greenberg \&
H.C. van de Hulst (Dordrecht:Reidel), 257

29. Soloman, P.M. \& Wrener, M.W. 1971, ApJ, 165, 49257

30. Spitzer, L., Drake, J.F., Jenkins, E.B., Morton, D.C., Rogerson, J.B., \& York, D,G. 1973, ApJ, 181, L116

31. va de Hulst, H.C. 1945, Ned. Tijd. Natuurkunde, 210

32. van de Hulst, H.C. 1948, Harvard Observatory Monographs, Centennial Symposia Dec. 1946, 73

33. van de Hulst, H.C., Muller, C.A., \& Oort, J.H. 1954, B.A.N., XII(452), 117

34. van de Hulst, H.C. 1997, Molecules in Astrophysics Half a Century Ago, in Molecules in Asrophysics: Probes and Processes, ed. E. van Dishoeck (Dordrecht: Kluwer), 13

35. Yoshida, N. Omukai, K., Hernquist, L, \& Abel, T. 2006, ApJ, 652, 6 